\title{
New Features in the Computational Infrastructure for Nuclear Astrophysics
}

\author{
Michael Smith ${ }^{* \dagger}$ \\ Physics Division, Oak Ridge National Laboratory \\ Oak Ridge, TN, 37831-6354, USA \\ E-mail: msmith@mail.phy.ornl.gov
}

\section{Eric J. Lingerfelt, Jason P. Scott, Caroline D. Nesaraja, Kyungyuk Chae}

Physics Division, Oak Ridge National Laboratory

Oak Ridge, TN, 37831-6354, USA

Dept. Physics \& Astronomy, Univ. of Tennessee

Knoxville, TN 37996-1200, USA

\section{Hiroyuki Koura}

Advanced Science Research Center, Japan Atomic Energy Agency

Tokai, Naka-gun, Ibaraki 319-1195, JAPAN

\section{Luke F. Roberts}

Physics Dept., Colorado College

14 E. Cache La Poudre

Colorado Springs, CO 80903, USA

\section{W. Raphael Hix, Daniel W. Bardayan, Jeffery C. Blackmon}

Physics Division, Oak Ridge National Laboratory

Oak Ridge, TN, 37831-6354, USA

\begin{abstract}
A Computational Infrastructure for Nuclear Astrophysics has been developed to streamline the inclusion of the latest nuclear physics data in astrophysics simulations. The infrastructure consists of a platform-independent suite of computer codes that are freely available online at http://nucastrodata.org. The newest features of, and future plans for, this software suite are given.
\end{abstract}

International Symposium on Nuclear Astrophysics - Nuclei in the Cosmos - IX

25-30 June 2006

CERN

\footnotetext{
* Speaker.

${ }^{\dagger}$ ORNL is managed by UT-Battelle, LLC, for the U.S. Department of Energy under contract DE-AC05-00OR22725.
} 


\section{General Description}

The Computational Infrastructure for Nuclear Astrophysics is a unique suite of computer codes, freely available online at nucastrodata.org, that is designed to greatly speed up the process of incorporating the latest nuclear physics results into astrophysical simulations and determining the astrophysical impact of these results. With a few mouse clicks, the suite enables creation, manipulation, and visualization of nuclear data sets as well as sample post-processing element synthesis calculations. The graphical interface enables Users to easily employ all, or just a few, of the features, and save and export their results. The suite facilitates an online community where large datasets can quickly be shared with others, comments exchanged, and consensus on best results reached. Reaction rates are stored in the format of the most widely used rate collection [1], while the nucleosynthesis calculations utilize the reaction network code of Hix \& Thielemann [2].

\section{Software Features}

The user-friendly interface enables Users to:

- upload their cross section or S factor data

- perform simple data evaluation tasks such as renormalizing or gain shifting

- extrapolate a cross section or S factor with a linear function or with a theoretical cross section dataset

- create reaction rates from cross sections and $\mathrm{S}$ factors

- calculate an inverse reaction rate by detailed balance

- scale up, down, or otherwise modify a reaction rate

- quickly find, plot, and compare all distinct rates for a given reaction

- generate reaction rates on a temperature grid and export

- create, access, merge, and manage libraries of reaction rates

- replace old rates in a library with newer versions

- automatically generate a "recipe" of how a library was created for future reference

- setup and run post-processing element synthesis calculations with new rate libraries

- compare simulations with different rate libraries

- vary input temperature / density histories and initial abundances in simulations

- plot simulation results - abundances as a function of time

- visualize the simulation results with $2 \mathrm{D}$ animated nuclide charts

- save your work in a dedicated disk space for future use

- share some or all of your work with colleagues around the world 


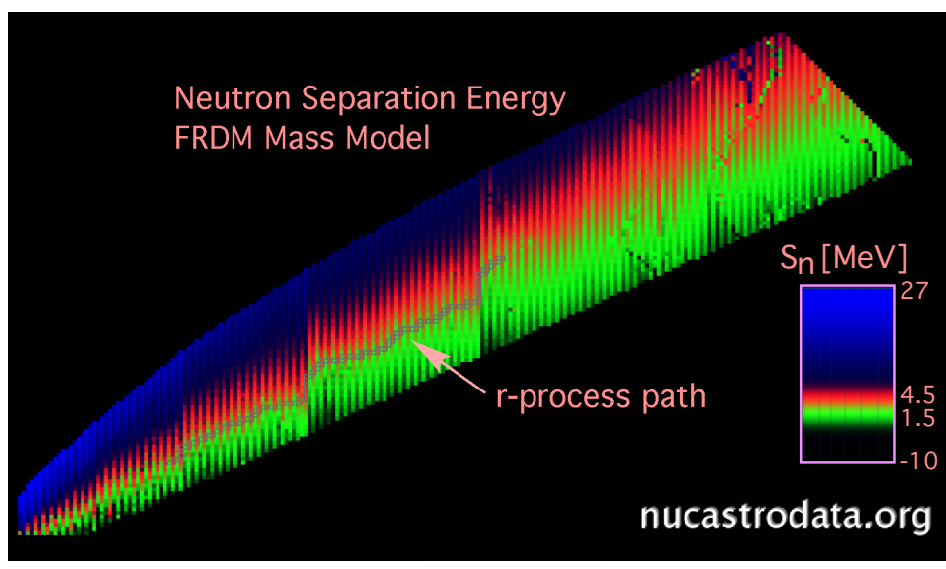

Figure 1: Neutron Separation Energy for the FRDM Mass Model

\section{Newest Features}

\subsection{Nuclear mass model evaluator}

This tool enables Users to examine theoretical nuclear mass models (their own or others) and to compare them with other models or with experimental mass data. 1D plots of mass difference and RMS mass difference as functions of Z, N, or A can be quickly generated. 2D nuclide charts of mass excess, Q-values, and separation energies (Figure 1) can be rapidly produced and customized in color mapping, parameter range, isotope labels, and zoom factor. The display of magic numbers, stable isotopes, and the r-process path can also be toggled on and off.

\subsection{Animation rendering}

This feature enables Users to render the animated nuclide charts displayed online for export and subsequent use in research and in presentations. Users can customize the desired frame rate and resolution, and a movie is rendered offline and the User notified of the download procedure.

\subsection{Simulation comparisons}

One of the most powerful features of the infrastructure is to run post-processing element synthesis calculations with different User-specified input reaction rate libraries and then quickly compare the results. Both direct comparisons (i.e., plotting the ratio of final abundances in two simulations) and indirect comparisons (i.e., running two sessions in parallel and comparing animations of abundance or reaction flux) are facilitated by this suite.

\subsection{Reaction rate searching and commenting}

In efforts to build and nurture an online community of reaction rate experts, Users can now read comments by others on their latest reaction rates and rate libraries and post their own comments. An email-type interface has been developed to facilitate the exchange of information on reaction rates. Another tool was developed to find and quickly display all rates for a given reaction that are found in different public and shared rate libraries. The combination of these tools enables 


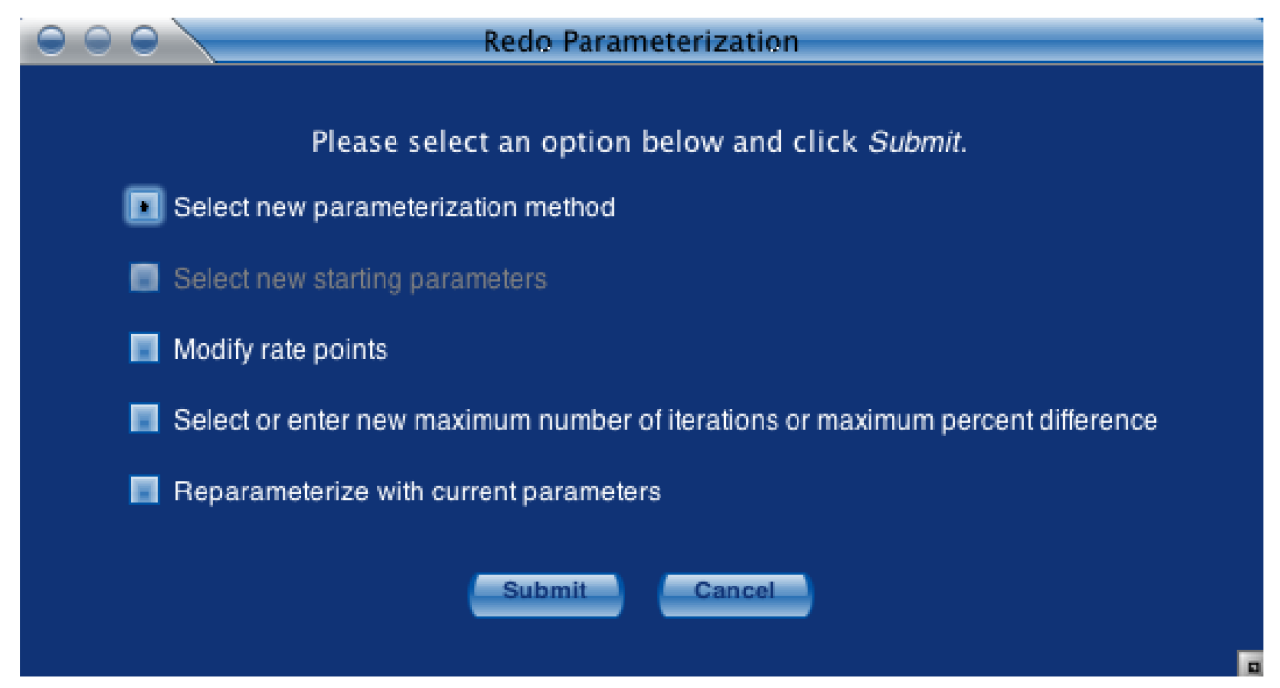

Figure 2: Control panel to redo reaction rate parameterizations

"recommended" rates to be more easily determined and a consensus among interested Users to be reached.

\subsection{Improved rate parameterization}

Users now have significantly more control over the program that fits pointwise reaction rates to analytic functions of temperature. They can now quickly redo parameterizations that are unsatisfactory by selecting a new method, selecting their own starting parameters for the fit, modifying the reaction rate points used in the fit, changing the iterations or desired fit accuracy, or simply continuing the fit using the best results from previous trial (Figure 2).

\subsection{New simulations}

Users can upload their element synthesis calculation results and utilize our suite of tools to display their results online and render their results in an offline animation. This has recently been done for $r$ process (Figure 3) and $v p$ process sample calculations. Uploaded simulations can be stored online and shared with others.

\subsection{Reaction flux and abundance derivative visualization}

Users can now quickly visualize the reaction flux via arrows from one isotope to another displayed on a nuclide chart. The thickness, pattern, flux range, and color mapping of the arrows are all easily customizable. Reaction flux can be shown for each time step of the simulation in an animated chart, or as a static chart showing integrated reaction flux overlaid with final synthesized abundances (Figure 4). Users can also display the time derivatives of nuclide abundances on an animated nuclide chart, which is especially useful to emphasize low abundance nuclei which are undergoing rapid abundance changes. 


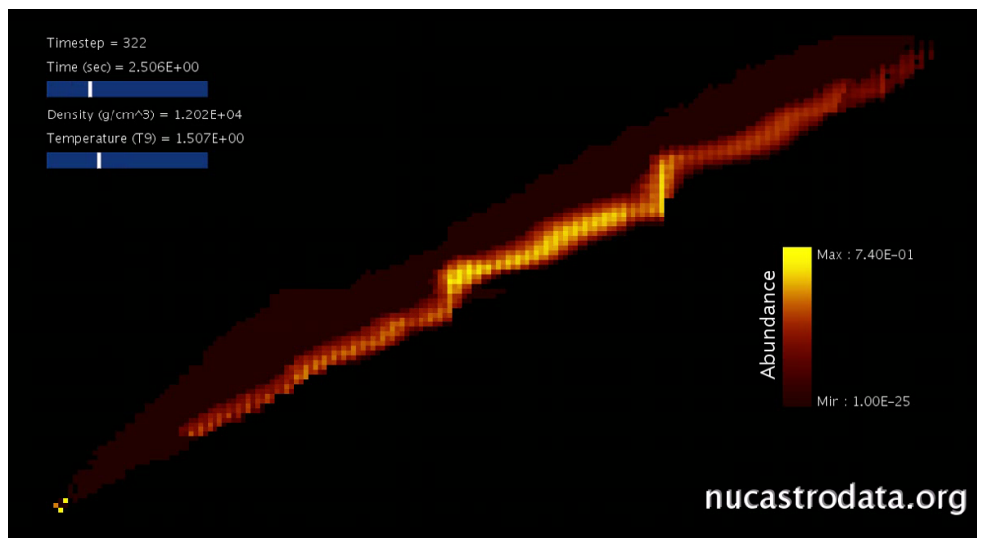

Figure 3: Abundances at one time-step in an $r$ process calculation

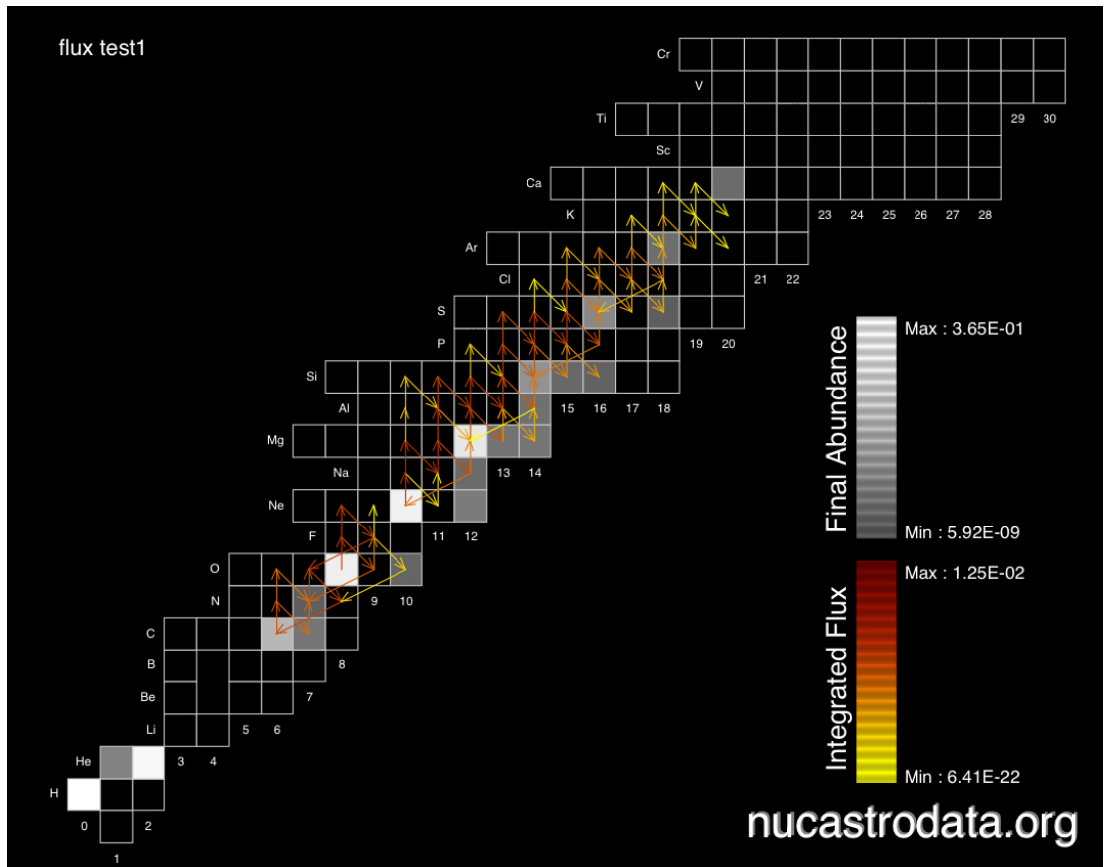

Figure 4: Integrated Reaction Flux and Final Abundances for a Nova Outburst

\section{Future Development}

Features planned to be added to this software suite include: rate calculations from nuclear resonance parameters; theoretical cross section calculations (using online mass models); additional simulation types (including $r$ process burning); additional interactivity in the mass model evaluator tool; and further enhancements of our visualization tools.

\section{References}

[1] T. Rauscher, F.-K. Thielemann, At. Data Nucl. Data Tables, 75, 1 - 351 (2000).

[2] W.R. Hix, F.-K. Thielemann, J. Comp. Appl. Math, 109, 321 (1999). 